\title{
Effect of a standardized treatment regime for infection after osteosynthesis
}

\author{
Pien Hellebrekers ${ }^{*}$ (D), Luke P. H. Leenen, Meriam Hoekstra and Falco Hietbrink
}

\begin{abstract}
Background: Infection after osteosynthesis is an important complication with significant morbidity and even mortality. These infections are often caused by biofilm-producing bacteria. Treatment algorithms dictate an aggressive approach with surgical debridement and antibiotic treatment. The aim of this study is to analyze the effect of such an aggressive standardized treatment regime with implant retention for acute, existing $<3$ weeks, infection after osteosynthesis.

Methods: We conducted a retrospective 2-year cohort in a single, level 1 trauma center on infection occurring within 12 months following any osteosynthesis surgery. The standardized treatment regime consisted of implant retention, thorough surgical debridement, and immediate antibiotic combination therapy with rifampicin. The primary outcome was success. Success was defined as consolidation of the fracture and resolved symptoms of infection. Culture and susceptibility testing were performed to identify bacteria and resistance patterns. Univariate analysis was conducted on patient-related factors in association with primary success and antibiotic resistance.

Results: Forty-nine patients were included for analysis. The primary success rate was $63 \%$ and overall success rate $88 \%$. Factors negatively associated with primary success were the following: Gustilo classification $(P=0.023)$, higher number of debridements needed $(P=0.015)$, inability of primary closure $(P=0.017)$, and subsequent application of vacuum therapy $(P=0.030)$. Adherence to the treatment regime was positively related to primary success $(P=0.034)$.
\end{abstract}

Conclusions: The described treatment protocol results in high success rates, comparable with success rates achieved in staged exchange in prosthetic joint infection treatment.

Keywords: Osteosynthesis, ORIF, Infection, Treatment, Osteomyelitis, Fracture

\section{Background}

Infection after surgical treatment of fractures is a complication with significant morbidity and in rare cases even mortality $[1,2]$. Consequences of infections include delayed or non-union of the fracture [3]. Infections that occur following osteosynthesis are typically caused by biofilm-forming bacteria, which adhere to the foreign body material [4]. After 3 weeks, a mature biofilm is formed, which makes it impossible to eradicate bacteria by antibiotics alone [5]. Most research in this field focuses on peri-prosthetic infection, despite of the different treatment challenges in prosthetic surgery and

\footnotetext{
*Correspondence: p.hellebrekers@umcutrecht.nl; hellebrekers.p@gmail.com Department of Surgery, University Medical Center Utrecht, P.O. Box 85500, 3508 GA Utrecht, The Netherlands
}

osteosynthesis. Treatment algorithms have been developed, which dictate aggressive debridement, antibiotic treatment, and if necessary staged replacement of the prosthetic material $[4,6]$.

Common treatment for peri-prosthetic infection consists of three pillars: surgical debridement, antibiotic therapy, and implant removal or staged change. However, in osteosynthesis, implant removal is unfavorable because of the recurrence of fracture instability which is related to protracted infection and inability to resolve infection $[7,8]$. This has consequences for the other aspects of treatment: as the implant stays in place, the biofilm does too. Surgical debridement can remove a gross part, but antibiotic therapy must be adjusted to the presence of a biofilm. 
For this reason, in our hospital, a standardized treatment regime was introduced, with the intention to treat the infection with implant retention until fracture healing is achieved. This regime consists of thorough surgical debridement, tissue cultures, and long-term antibiotic combination therapy with rifampicin. Rifampicin was chosen because of its high bioavailability and penetration into biofilms [9].

Soft tissue damage and wound coverage causes additional difficulties for the healing process during infection after osteosynthesis. For the latter, flap cover or vacuum therapy are the preferred choices within our hospital.

The aim of this study was to analyze the success rate of patients with infection after osteosynthesis undergoing surgical debridement, tissue cultures, and long-term antibiotic combination therapy with rifampicin.

\section{Methods}

\section{Study design and population}

This was a retrospective cohort study, with a level of evidence therapeutic level IV. Patients treated for early and delayed (<12 months) infection after osteosynthesis in a level 1 trauma center were analyzed [4]. Included patients were between 18 and 80 years of age and treated for infection following osteosynthesis from the introduction of the regime in January 2011 until September 2013. Subjects were identified in the operation register. All acute infections following osteosynthesis were included, independent of localization, implant used, or Gustilo classification. Infections following prosthetic surgery and revision arthroplasties were excluded. Subjects in whom symptoms (e.g., redness, drainage from surgical wound, fever, pain, elevated CRP, or leukocytes) had existed for longer than 3 weeks, thus with matured biofilm, were excluded [5]. Also, patients with consolidated fractures at presentation of infection were excluded, here implant removal was considered to be the appropriate treatment. Patient characteristics were collected from electronic patient files. Extracted data are listed in the baseline characteristics.

\section{Treatment}

All patients included in the study were treated by initial thorough surgical debridement of affected soft tissue and necrotic bone during which deep tissue cultures were obtained. Surgical debridement was repeated as often as needed. When primary closure could not be performed, continuous applied vacuum therapy (VAC ${ }^{\circ}$ System) was applied to provide temporary covering and improve secondary wound healing. Small, non-infected skin defects are left untreated but are considered "open" in this study with respect to time to closure. In case of large soft tissue damage, skin transplant or free flaps were to be considered. Only when infection persisted despite multiple surgical debridements without the prospect of infection control, implant removal was considered, and alternative form of stability at the fracture site was initiated.

Antibiotic combination therapy started immediately after the first surgical intervention and consisted of 10 days of intravenous (i.v.) vancomycin and rifampicin (Fig. 1). Vancomycin was the agent of choice for empirical therapy because of its activity against a broad spectrum of microorganisms, high incidence of Gram positive (e.g., Staphylococcus aureus) infections, and the synergetic effect with rifampicin [10-13]. Vancomycin therapy was started as twice daily $1000 \mathrm{mg}$ i.v. and was adjusted to maintain serum levels between 15 and $20 \mathrm{mcg} / \mathrm{ml}$. Rifampicin was given twice daily $450 \mathrm{mg}$ i.v. Rifampicin was added because of its relatively easy penetration into biofilm, its high bioavailability, and its ability to affect organisms in stationary growth phase [9, 14-19]. When tissue cultures grew bacterial pathogens and susceptibility data were available, vancomycin therapy was switched to another, narrower spectrum, antibiotic as indicated by the treating physician. Rifampicin was continued unless rifampicin-resistant bacteria were found. After the intravenous administration period, oral combination antibiotic therapy with rifampicin was continued for ten additional weeks [4].

Treatment was considered to be in accordance with the regime if there were no or minor deviations, in line with clinical practice. Here, minor deviation was defined as deviation of duration of antibiotic therapy or when tissue or swab cultures were not adequately obtained. Major deviation from the regime consisted of no surgical debridement, initial implant removal, no or inadequate oral or intravenous antibiotic therapy, or monotherapy with rifampicin.

Subjects were followed in the outpatient clinic for at least 3 months or until fracture consolidation and infection control were achieved.

\section{Patient identification}

In total, 80 patients with infection or osteomyelitis after osteosynthesis were identified. Twenty-one patients were excluded after thorough review (Fig. 2). Patients who have had symptoms for over 3 weeks (not acute) were excluded from final analysis $(n=10)$. The remaining 49 $(60 \%)$ patients were eligible for analysis (Table 1$)$. The median age is 45 years (range 18-69), and the majority is of male gender (76\%). Infection was mainly of pelvic ring, femur, and tibia (67\% combined), and in $11(22 \%)$ cases, it concerned complicated fractures (Gustilo IIIA-IIIC). Plates and nails were the most often used implants. Ninety percent of the infections manifested in the first 3 months after osteosynthesis or last surgery related to the trauma on the respective body part. Overall, adherence to the 


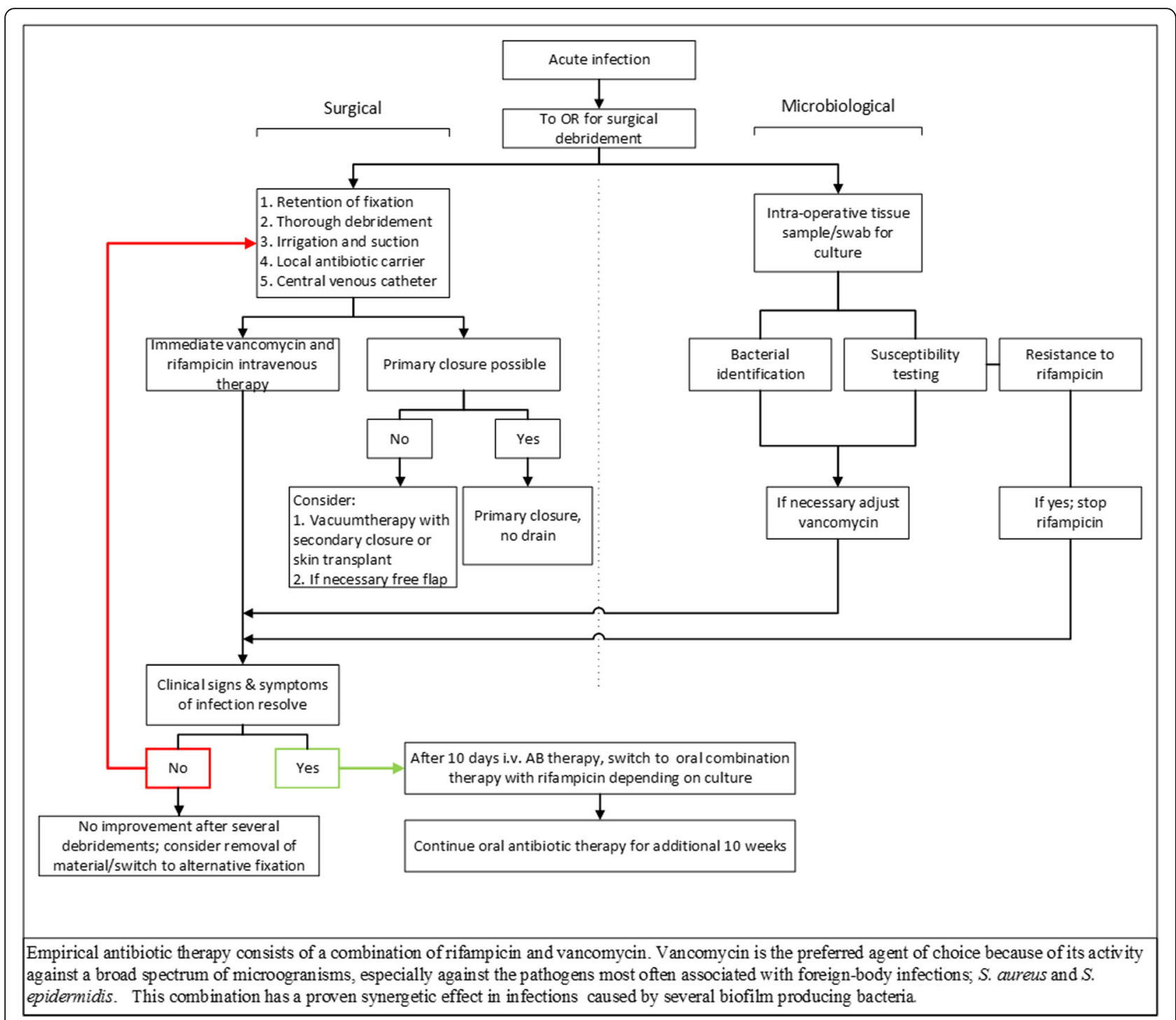

Fig. 1 A flow scheme of the standardized treatment regime as executed in our hospital

regime increased over time from $75 \%$ at the time of introduction to $100 \%$ in 2013 (Table 1).

\section{Microbiology}

From all subjects, peri-operative cultures (tissue, swab, fluid) were obtained during initial surgical debridement and were repeated with every additional surgery. Isolates were cultured and identified using standard techniques until growth and identification was apparent or to a maximum of 6 days in case of negative culture. Susceptibility testing was performed on isolates using Phoenix automated susceptibility testing, disk diffusion, and/or E-test.

\section{Study endpoints}

The primary endpoint was success. Primary success was defined as consolidation of the fracture on X-ray and clinical examination, as assessed by the treating physician, resolved clinical signs and symptoms of infection after completion of the standardized treatment regime. Consolidation is defined as ossified callus bridging $\geq 3$ cortices on radiography and stable and painless bone at attempted angulation on examination. Since this was a retrospective analysis with a variety of types of fractures and fracture sites, covering all grades of the AO- and Gustilo classification, no strict timeframe or routine laboratory follow-up was available. Secondary success was defined as consolidation of the fracture with no signs of infection after a prolongation of the standardized treatment regime or delayed implant removal (Fig. 1). Time to secondary success was described. Any other outcome than primary or secondary success was considered treatment failure. A univariate analysis was performed to 


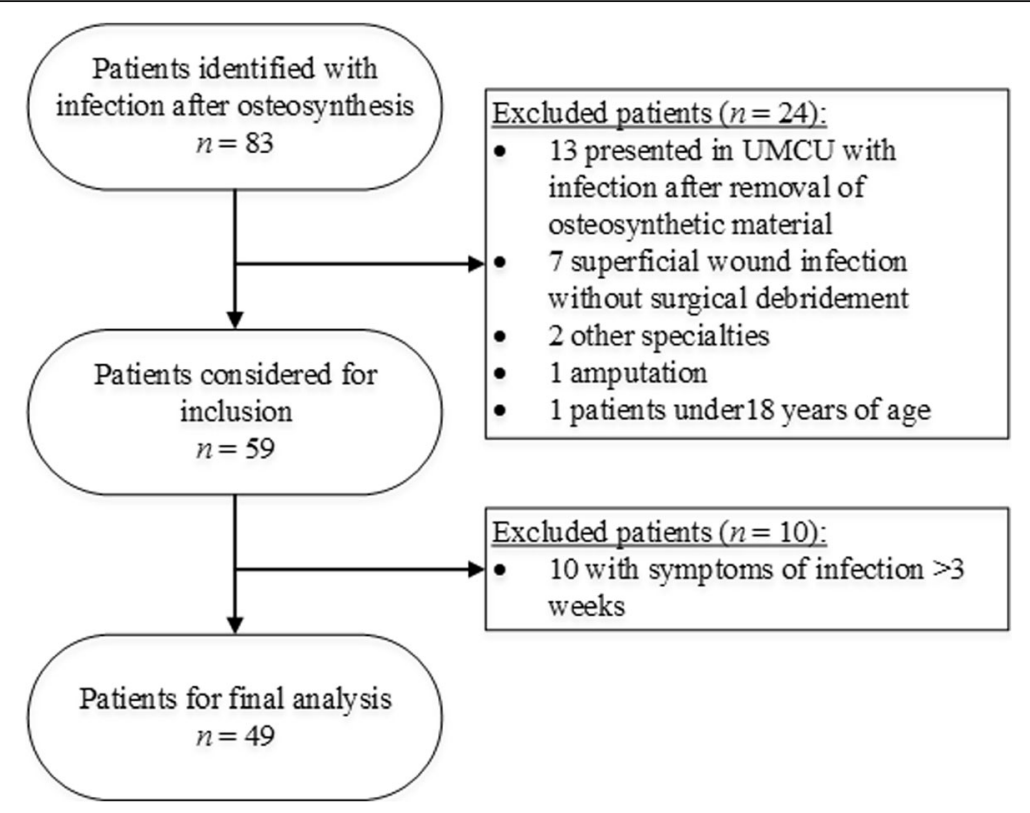

Fig. 2 Patient identification and inclusion

identify factors associated with treatment failure, e.g., type of fracture, variation in treatment (surgical and/or microbial), and patient characteristics. Overall success was the primary and secondary success combined.

The microbial characteristics of the infections, the proportion of infections caused by vancomycin- and rifampicin-susceptible microorganisms, and possible factors influencing incidence of resistance were identified.

\section{Statistical analysis}

Primary endpoints, primary and secondary success, were descriptive. Concerning secondary endpoints, nonparametric test were applied. A univariate analysis was conducted. Due to the limited number of cases, a multivariate analysis was not applicable. In the univariate analysis, association was determined using Fisher's exact test for dichotomous variables, linear-by-linear for nominal, and Mann-Whitney $U$ for continuous data. $P$ values $<0.05$ are considered as significant.

\section{Results}

\section{Study population}

Primary closure after initial debridement could be performed in $47 \%$ of the patients. In the remaining, closure was achieved through secondary wound healing with or without vacuum therapy, skin or free flap transplantation. Time to delayed skin closure, including small communicating defects despite transplantation, demonstrated a median closure time of 85 days, with outliers up to 540 days. Forty-seven patients were treated with intravenous combination therapy, for a median duration of 10 days, range 3-116 days. The 2 patients who did not receive intravenous antibiotic therapy did not demonstrate signs of infection surrounding the implant during surgical debridement or infection had not reached the fracture site. The decision was made to treat as superficial wound infection. Of the patients who did not get rifampicin, 4 had implant removal, 1 had only one screw in situ, 2 patients had the entire antibiotic therapy ceased because of low suspicion of deep infection. In 1 patient, rifampicin therapy was stopped because of interaction with psychiatric drugs. The entire antibiotic therapy had duration with a median of 80 days, ranging from 0 to 126 days. Antibiotic agents used in the intravenous combination therapy were most often vancomycin, rifampicin, and ciprofloxacin. Oral agents were predominantly rifampicin and flucloxacillin.

\section{Success}

Primary success after completion of standardized treatment regime was reached in $63 \%$ of the patients after a median duration of 4 months (range 1-13 months). Of the patients who primarily succeeded, $90 \%$ was treated according to the regime or had only minor deviation. Statistical significance with a negative association toward the primary success group were found for higher Gustilo classification $(\geq \mathrm{II})(P=0.023)$, higher number of debridements performed $(P=0.015)$, impossibility of primary closure $(P=0.017)$, and in line with this the necessity for vacuum dressing after debridement $(P=0.030)$. A statistical significant positive association was found when patients were treated according to the regime $(P=0.025)$. A shorter duration of oral antibiotic therapy $(P=0.033)$ than specified in the standardized treatment regime was negatively related (Table 1 ). 
Table 1 Baseline characteristics and the association with primary cure

\begin{tabular}{|c|c|c|c|c|}
\hline \multirow[t]{2}{*}{ Variable } & \multirow{2}{*}{$\begin{array}{l}\text { Baseline } \\
(n=49)\end{array}$} & \multicolumn{2}{|c|}{ Primary success rate } & \multirow[t]{2}{*}{$P$ value } \\
\hline & & No $(n=18)$ & Yes $(n=31)$ & \\
\hline \multicolumn{5}{|l|}{ Patient } \\
\hline Age, years & $45(18-69)$ & $47(25-66)$ & $45(18-69)$ & 0.194 \\
\hline Male gender & 37 & 13 & 24 & 0.738 \\
\hline Smoking & 20 & 10 & 10 & 0.226 \\
\hline \multicolumn{5}{|l|}{ Comorbidities } \\
\hline Diabetes mellitus & 5 & 1 & 4 & 0.639 \\
\hline Psychiatric disorder & 14 & 6 & 8 & 0.744 \\
\hline BMl & $26.3(19.5-41.8)$ & $26.3(19.5-41.8)$ & $26.3(20.2-38.5)$ & 0.719 \\
\hline Oral corticosteroid use & 1 & - & 1 & 1.0 \\
\hline Follow-up, months & $12(3-36)$ & $19(8-32)$ & $10(3-36)$ & 0.008 \\
\hline \multicolumn{5}{|l|}{ Fracture } \\
\hline Localization & & & & 0.140 \\
\hline Sternum/costa & 2 & 1 & 1 & \\
\hline Humerus & 1 & - & 1 & \\
\hline Radius/ulna & 4 & 2 & 2 & \\
\hline Pelvic ring & 11 & 2 & 9 & \\
\hline Femur & 8 & 3 & 5 & \\
\hline Tibia/fibula & 14 & 8 & 6 & \\
\hline Ankle & 6 & - & 6 & \\
\hline Foot & 3 & 2 & 1 & \\
\hline Type & & & & 0.023 \\
\hline Closed & 28 & 6 & 22 & \\
\hline \multicolumn{5}{|c|}{ Open Gustilo classification } \\
\hline । & 6 & 2 & 4 & \\
\hline$\|$ & 3 & 2 & 1 & \\
\hline$\| \mathrm{A} A$ & 4 & 4 & - & \\
\hline IIIB & 4 & 1 & 3 & \\
\hline IIIC & 3 & 2 & 1 & \\
\hline AO classification & & & & 0.144 \\
\hline A & 4 & 0 & 4 & \\
\hline B & 13 & 4 & 9 & \\
\hline$C$ & 25 & 10 & 15 & \\
\hline Not applicable & 7 & & & \\
\hline Type of osteosynthesis & & & & 0.649 \\
\hline (Cerclage) wires & 1 & - & 1 & \\
\hline Screws & 6 & 2 & 4 & \\
\hline Nail & 12 & 6 & 6 & \\
\hline Plate & 30 & 10 & 20 & \\
\hline
\end{tabular}

Data are presented as the number of cases with the percentage in parenthesis or as median with the range in parenthesis i.v. intravenous

$P$ values of $<0.05$ are considered significant. Only $P$ values of $<0.05$ are in italic

Secondary success was achieved in 12 of the 18 remaining patients in whom success was not reached after completion of a single run. Secondary success was achieved after a median duration of 14 months (range
6-24 months). Of those with secondary success, 11 patients demonstrated persisting or relapsing symptoms of inflammation at the infection site before consolidation occurred. Four were treated with implant removal and 
repeated antibiotic therapy, 4 only with implant removal, and 3 only with repeated antibiotic therapy. Only in 1 patient the reason of primary failure was non-union of the fracture, in which success was reached after revision osteosynthesis. When primary and secondary success rates were combined, this results in an overall success rate of $88 \%$ (Table 2).

Of the patients in whom success was not reached, all but one had diminished signs and symptoms of infection, yet without consolidation of the fracture. One patient still had persisting infection at the moment of analysis (Table 3 ).

\section{Microbiology}

Of the 49 included cases, in one case, no tissue samples were obtained for culture and 5 had shown no growth at first culture, despite intraoperative signs of infection. In
16 of the cases, only $S$. aureus could be cultured from the samples retrieved, in all but one case it concerned methicillin-susceptible $S$. aureus. In 2 cases, solely coagulase-negative staphylococci (CoNS), and in 4 cases, other single organisms were cultured (Enterococcus faecalis, Streptococcus dysgalactiae, Enterobacter cloacae complex, Enterobacter gergoviae) (Table 1). In the remaining 21 cases, culture showed mixed microorganisms, of which in $48 \%$ it included S. aureus, all methicillin susceptible. Based on first culture, the choice of empirical treatment with rifampicin and vancomycin was adequate in 88 and $91 \%$ of the cases, respectively (Table 4).

\section{Vancomycin and rifampicin resistance}

No vancomycin resistance was demonstrated in any of the cases. Rifampicin resistance at first culture was

Table 2 Infection and treatment characteristics and association with primary cure

\begin{tabular}{|c|c|c|c|c|}
\hline \multirow[t]{2}{*}{ Variable } & \multirow{2}{*}{$\begin{array}{l}\text { Baseline } \\
(n=49)\end{array}$} & \multicolumn{2}{|c|}{ Primary success rate } & \multirow[t]{2}{*}{$P$ value } \\
\hline & & No $(n=18)$ & Yes $(n=31)$ & \\
\hline \multicolumn{5}{|l|}{ Infection } \\
\hline Type of infection & & & & 0.260 \\
\hline Early & 44 & 15 & 29 & \\
\hline Delayed & 5 & 3 & 2 & \\
\hline \multicolumn{5}{|l|}{ Microorganism } \\
\hline Negative culture & 5 & 1 & 4 & 0.623 \\
\hline Staphylococcus aureus & 16 & 6 & 10 & 1.0 \\
\hline CoNS & 2 & 1 & 1 & 1.0 \\
\hline Polymicrobial culture & 21 & 10 & 11 & 0.206 \\
\hline Other single microorganism & 4 & - & 4 & 0.282 \\
\hline Rifampicin resistance at first culture & 3 & 2 & 1 & 0.544 \\
\hline Rifampicin resistance in all cultures & 8 & 5 & 3 & 0.250 \\
\hline \multicolumn{5}{|l|}{ Treatment } \\
\hline Number of debridements performed & $2(0-14)$ & $3(0-14)$ & $2(1-5)$ & 0.015 \\
\hline \multicolumn{5}{|l|}{ Closure } \\
\hline Primary closure & 23 & 4 & 19 & 0.017 \\
\hline VAC therapy after debridement & 17 & 10 & 7 & 0.030 \\
\hline Time to closure after debridement, days & $157(8-700)$ & $271(38-700)$ & $149(8-157)$ & 0.000 \\
\hline \multicolumn{5}{|l|}{ Antibiotic therapy } \\
\hline Duration i.v. antibiotic therapy & $10(0-116)$ & $14(0-77)$ & $10(0-116)$ & 0.203 \\
\hline Duration oral antibiotic therapy & $70(0-112)$ & $63(0-89)$ & $70(0-112)$ & 0.033 \\
\hline Duration total antibiotic therapy & $80(0-126)$ & $80(0-126)$ & $81(9-116)$ & 0.186 \\
\hline Duration of rifampicin therapy & $80(5-116)$ & $75(6-97)$ & $80(5-116)$ & 0.118 \\
\hline NSAID use during treatment & 36 & 14 & 31 & 0.743 \\
\hline Treated according to protocol & & & & 0.025 \\
\hline Yes & 39 & 11 & 28 & \\
\hline No & 10 & 7 & 3 & \\
\hline
\end{tabular}

Data are presented as the number of cases with the percentage in parenthesis or as median with the range in parenthesis i.v. intravenous

$P$ values of $<0.05$ are considered significant. Only $P$ values of $<0.05$ are in italic 
Table 3 Overview of patients without success

\begin{tabular}{|c|c|c|c|c|c|c|c|}
\hline Patient no. & Follow-up (mo) & Fracture, Gustilo & Closure & $\mathrm{MB}$ & $\begin{array}{l}\text { Rifampicin } \\
\text { resistance }\end{array}$ & Treatment & Failure reason \\
\hline 1 & 16 & Distal tibia/fibula, IIIA & Free flap & PM & No & $\begin{array}{l}\text { Multiple surgical debridement, } \\
\text { local antibiotic treatment, } \\
\text { revision osteosynthesis, } \\
2 \times \text { protocol treatment }\end{array}$ & Amputation necessary \\
\hline 2 & 24 & Pelvic ring, IIIC & Free flap & PM & Yes & $\begin{array}{l}\text { Removal OSM at initial } \\
\text { debridement, multiple surgical } \\
\text { debridement, local antibiotic } \\
\text { treatment }\end{array}$ & $\begin{array}{l}\text { Non-union, continuous soft } \\
\text { tissue problems }\end{array}$ \\
\hline 3 & 16 & Distal femur, IIIC & $\begin{array}{l}\text { Split skin } \\
\text { graft }\end{array}$ & PM & No & $\begin{array}{l}\text { Multiple surgical debridement } \\
\text { and antibiotic therapy, local } \\
\text { antibiotic therapy, removal OSM }\end{array}$ & $\begin{array}{l}\text { Non-union, persisting low } \\
\text { grade infection }\end{array}$ \\
\hline 4 & 15 & Shaft tibia/fibula, IIIB & Free flap & CoNS & Yes & $\begin{array}{l}\text { Masquelet technique, surgical } \\
\text { debridement, no intravenous } \\
\text { antibiotic therapy, when relapse } \\
\text { adequate protocol treatment }\end{array}$ & Non-union \\
\hline 5 & 10 & Distal femur, I & Primary & NG & NA & $\begin{array}{l}\text { Infection after re-osteosynthesis } \\
\text { for pseudo-arthrosis, single } \\
\text { surgical debridement, perfect } \\
\text { protocol treatment }\end{array}$ & Non-union \\
\hline 6 & 11 & $\begin{array}{l}\text { Shaft tibia/fibula, } \\
\text { unknown }\end{array}$ & $\begin{array}{l}\text { Split skin } \\
\text { graft }\end{array}$ & PM & No & $\begin{array}{l}\text { Infection after sequestrectomy } \\
\text { and re-osteosynthesis for } \\
\text { pseudo-arthrosis, multiple surgical } \\
\text { debridement and antibiotic } \\
\text { treatment. }\end{array}$ & $\begin{array}{l}\text { Non-union, persisting } \\
\text { wound }\end{array}$ \\
\hline
\end{tabular}

mo months, MB microbiology, PM polymicrobial, CoNS coagulase-negative Staphylococci, NG no growth, OSM osteosynthetic material, NA not applicable

demonstrated in $3(6 \%)$ cases, all CoNS, one proven to be Staphylococcus epidermidis. In 18 cases, no follow-up tissue sample or swabs were taken. Twenty-two out of the 30 follow-up cultures grew microorganisms. Rifampicin resistance was demonstrated in 7 cultures, of which 2 overlapped with resistance at first culture. In the 5 new cases, $S$. aureus (methicillin-susceptible $S$. aureus; MSSA) was seen once, and in 4 cases, $S$. epidermidis was demonstrated.

Rifampin resistance was significantly more frequently found after secondary than after primary closure of the wound $(P=0.044)$ (Table 3$)$. Secondary closure was associated with longer duration of intravenous antibiotic therapy $(P=0.008)$. No association was found between rifampicin resistance and primary $(P=0.250)$ or overall success $(P=0.580)$ (Table 5$)$.

\section{Discussion}

The aim of the study was to evaluate the effect of a standardized treatment regime for acute infection after osteosynthesis. Results demonstrate a high success rate, especially when patients are treated strictly according to the regime or with only minor deviations, resulting in an overall success rate of $88 \%$. In almost all patients,

Table 4 Microbiological characteristics of included cases

\begin{tabular}{|c|c|c|c|c|c|}
\hline \multirow[t]{2}{*}{ Microorganism } & \multirow[t]{2}{*}{ Cases $(n=49)$} & \multirow{2}{*}{$\begin{array}{l}\text { Primary success } \\
\text { rate }(\%)\end{array}$} & \multirow{2}{*}{$\begin{array}{l}\text { Overall success } \\
\text { rate }(\%)\end{array}$} & \multicolumn{2}{|c|}{ Antimicrobial susceptibility ${ }^{\mathrm{a}}(\%)$} \\
\hline & & & & Rifampicin & Vancomycin \\
\hline No growth & $5(10 \%)$ & 80 & 80 & - & - \\
\hline Staphylococcus aureus & $16(33 \%)$ & 63 & 88 & 100 & 100 \\
\hline MSSA & $15(94 \%)$ & & & & \\
\hline MRSA & $1(6 \%)$ & & & & \\
\hline Coagulase-negative staphylococci & $2(4 \%)$ & 50 & 100 & 100 & 100 \\
\hline Polymicrobial infections ${ }^{b}$ & $21(43 \%)$ & 52 & 86 & 86 & 91 \\
\hline Other single microbial infections ${ }^{c}$ & $4(8 \%)$ & 100 & 100 & 25 & 50 \\
\hline Rifampicin resistance at first culture & $3(6 \%)$ & Overall susceptibility & & 88 & 91 \\
\hline
\end{tabular}

MSSA methicillin-susceptible Staphylococcus aureus, MRSA methicillin-resistant Staphylococcus aureus

${ }^{a}$ Empirical therapy is considered adequate when first culture obtains one or more susceptible pathogenic microorganisms

${ }^{b} 10$ cultures contained $S$. aureus among others

'Single microorganisms were cultured, concerning Streptococcus dysgalactiae, Enterobacter cloacae complex, Enterobacter gergoviae, and Enterococcus faecalis 
Table 5 Association between closure after surgical debridement and overall rifampicin resistance

\begin{tabular}{llll}
\hline & \multicolumn{2}{c}{ Overall rifampicin resistance } & $P$ value \\
\cline { 2 - 3 } & No $(n=31)$ & Yes $(n=8)$ & \\
\hline $\begin{array}{llll}\text { Closure after surgical } \\
\text { debridement }\end{array}$ & 18 & 1 & 0.044 \\
$\quad$ Primary closure & 13 & 7 & \\
$\quad$ Secondary closure or free flap & $127(38-540)$ & 117 & 0.253 \\
$\begin{array}{l}\text { Time to closure }{ }^{\text {a }} \text {, median } \\
\text { days (range) }\end{array}$ & 20 & 3 & 0.250 \\
Primary success & 28 & 6 & 0.580 \\
\hline Overall success & 20
\end{tabular}

${ }^{\mathrm{a} C a s e s}$ with primary closure are excluded

$P$ values of $<0.05$ are considered significant. Only $P$ values of $<0.05$ are in italic

implants could be retained until fracture consolidation was achieved. We found a negative association between the achievement of primary success and Gustilo classification, necessary number of debridements, inability of primary closure after debridement, and subsequent application of vacuum therapy. In case of treatment failure, all but one subject had infection control, but lacked fracture consolidation, one case had a persisting infection at the moment of analysis.

There are only a limited number of studies on the outcome after combination therapy with rifampicin for infection after osteosynthesis in an in vivo setting. Data available for comparison mostly refer to prosthetic joint infection (PJI). The reported success rate of PJI treated with antibiotic combination therapy with rifampicin varies widely. Zimmerli et al. as well as Barberan et al. and Drancourt et al. studied infection following osteosynthesis and the effect of antibiotic combination therapy with rifampicin [20-22]. They analyzed both PJI and osteosynthesis treated with initial implant retention and combination antibiotic therapy and found a success rate of $48 \%$ after an average follow-up of 23.5 months. The study of Barberan et al. solely included patients with osteosynthesis and found a success rate of $72 \%$ [21]. Notably, they only performed surgical debridement in $72 \%$ of the cases. In contrast, Zimmerli et al. studied rifampicin combination therapy for infection associated with orthopedic implants, combining prosthetic surgery, and osteosynthesis and showed a $100 \%$ success rate in the rifampicin combination group [20]. Additionally, a recent study showed high success rates (90\%) with the use of rifampicin in staphylococci-positive infections. In contrast with our study, they handled strict selection criteria for patients to be treated according to their algorithm, whereas we included all patients in spite of the condition of the soft tissue or found pathogens [23]. Other treatment regimens for infection following osteosynthesis with retention of the implant are proposed without rifampicin combination therapy. For example by Aytac et al., they propose a treatment containing of thorough surgical debridement with insertion of a persisting fistula and antibiotic treatment (4-8 weeks). They accomplish comparable promising success rates. However, they excluded subjects with mild to extensive soft tissue injury, sepsis, and re-occurrence of infection $>1$ year. Whereas we included all acute infections to optimize the reflection of current clinical practice [24].

We carried out a regimen in which implant retention was one of the objectives for all patients. In contrast, treatment guidelines for PJI only consider patients who are presented within 30 days after prosthesis implantation for retention of prosthesis strategy [6]. Implant removal is not desirable in case of acute infection as osteosynthesis serves two different goals. First, the stability achieved by fixation is critical for fracture healing [22, 25]. When conditions are created in which (micro-)motion between bone fragments is possible, resorption and necrosis of affected bone will occur [8]. Secondly, the aim of operative fracture management and early mobilization is to prevent loss of function due to scarring of the surrounding soft tissue or joint stiffness [26]. Special consideration should be given to infection after intramedullary fixation, the common stand is that diagnosis of the infected medulla and eradication of the infection is not feasible without implant exchange (Fig. 3) [27]. However, no international consensus is reached and other studies describe good cure rates with retention of the implant and propose treatment algorithms with nail retainment in early and delayed infections [28]. In this study, we subjected these patients to the same regime and we aimed to retain the implant in all. If the implant is to be removed, immobilization of the affected body part is required, with loss of function as a result [26]. Whereas removal or exchange of the implant provides the opportunity to remove the biofilm and as a result significantly reduce the bacterial load, and in case of implant retention, the surgical debridement and antibiotic therapy play a more important role. Combined, these different approaches make it difficult to compare outcome of infection after osteosynthesis with outcome after prosthetic joint infection.

Rifampicin is a potent antibiotic for the treatment of infections with biofilm-forming bacteria, which is a common cause for infection after osteosynthesis. The consequence of the increasing use of rifampicin is the emergence of resistance to this antibiotic and the occurrence of cross-resistance $[11,29]$. In vivo studies give indications for emergence of resistance in cases with inadequate surgical debridement and/or high bacterial load [5]. We found a significant difference in rifampicin resistance between patients with primary closure following surgical debridement and patients with secondary closure, skin transplant, or free flap, with a higher frequency of resistance in the latter. This can be explained 


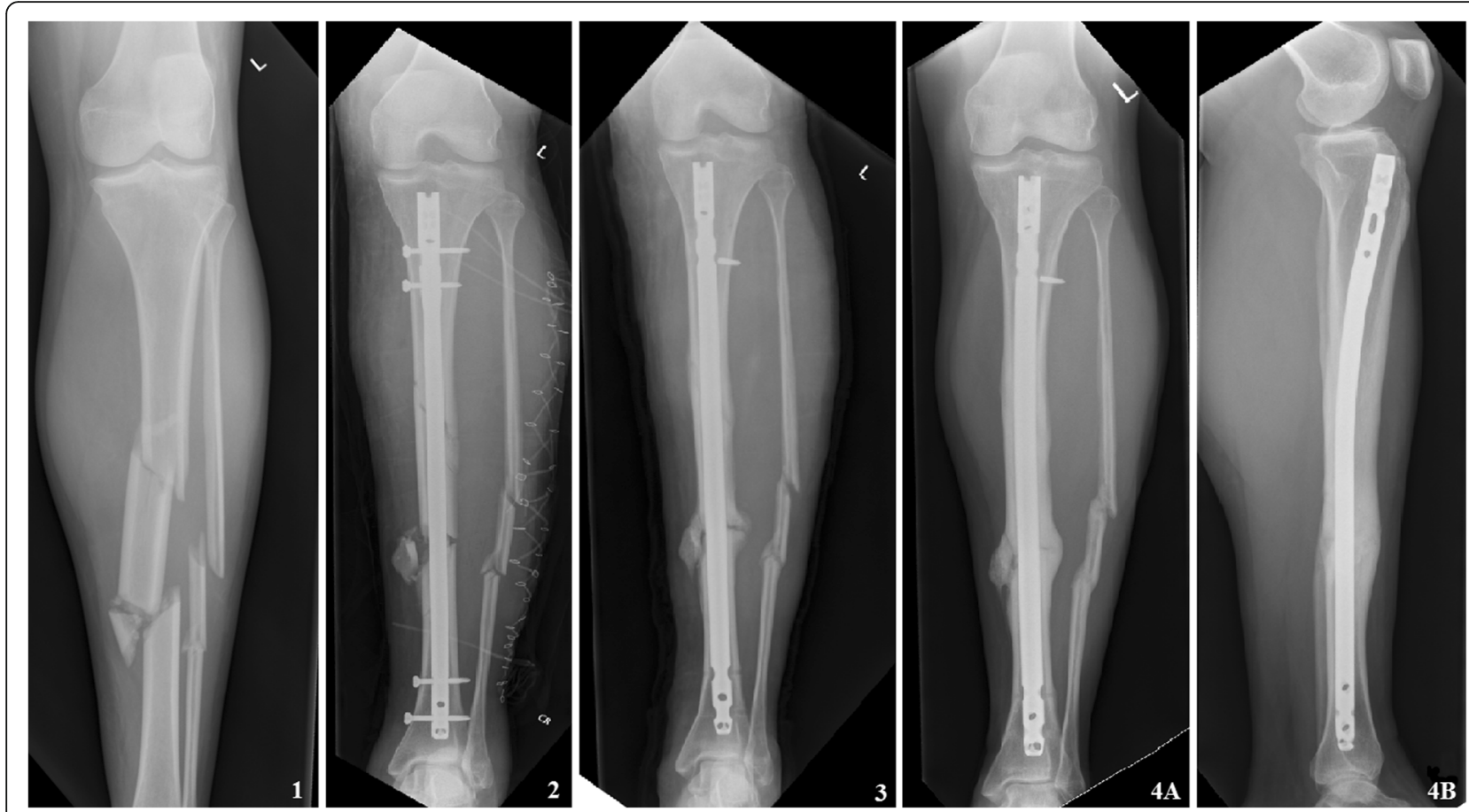

Fig. 3 Example of an infection after intramedullary osteosynthesis for a tibial shaft fracture (AO42-C2). 1 At presentation at the emergency department. $\mathbf{2}$ After nailing, the fracture was accompanied by a compartment syndrome treated with fasciotomy. $\mathbf{3}$ Patient presented on the emergency department with redness, swelling, and elevated CRP and leukocytes 7 months after initial osteosynthesis. The patient was admitted, and a thorough surgical debridement was performed immediately, the nail was left in situ. Intraoperative tissue cultures were obtained, and empirical antibiotic combination therapy was started. 4 Six months later, the fracture showed bridging of three cortices on X-ray and all signs and symptoms of infection were diminished. The patient visited the outpatient clinic 2 years later for an unrelated issue. The leg was entirely healed without pain with full weight bearing and full range of motion. The nail was never removed

by the fact that open wounds are constantly recontaminated with (commensal) skin flora and there is a selection of, intrinsically, resistant mutants.

Another factor that can be of significance with regard to wound- and bone healing in our cohort is the presence of comorbidities. Although this typical trauma cohort consist mainly of young, psychically healthy adults, comorbidities such as psychiatric disorders and characteristics as smoking were abundant and may have contributed to impaired healing [30, 31].

Furthermore, having an open wound or bad soft tissue condition may maintain infection, prolonging the necessity for antibiotic therapy; and thereby, the exposure of microorganisms to rifampicin and emergence to rifampicin becomes more opportune. However, in this study, total duration of the administration of rifampicin did not differ between the primary and secondary closure group.

The limitations to this study were inherent to the retrospective character. There was neither the aimed homogeneity in treatment approach, nor in follow-up or strictly defined objective outcome measures (e.g., laboratory data, time frame). Due to the limited number of cases included, a multivariate analysis could not be conducted. Thereby, no statements could be made on independent risk factors for initial failure of the standardized treatment regime.

Additionally, we included all acute infections after osteosynthesis, creating a heterogeneous group of Gustilo classification, affected body part, and choice of internal fixation adding up to the difficulty to assign results to specific elements. However, considering the low overall incidence of $1-2 \%$ for infection following osteosynthesis, it is not feasible to form a cohort with a homogeneous population, concerning fracture and implant type.

Another drawback is the fact that there was a fair amount of variation within the treatment regime. However, we wanted to show the real-time results of a standardized treatment, and in that way present current practice.

Due to the fact that we analyzed a single cohort from one, level 1 trauma center, one may argue that the results found in this study could not be generalized. Yet, since we are a specialized center, also patients from level 2 and 3 centers were referred and included. In addition, open fractures, seen more in multi-trauma patients, are known to be at higher risk to develop infection. 
Moreover, lacking a control group, no comparison could be drawn with results in patients treated according to a different protocol. However, this is the first series to describe outcome of standardized aggressive treatment for infection after osteosynthesis, consisting of implant retention, thorough surgical debridement, and intensive antibiotic combination therapy with rifampicin.

\section{Conclusions}

This study demonstrates an acceptable success rate in a clinical challenging problem of infection after osteosynthesis by a standardized treatment regime using aggressive surgical debridement and immediate broad combination antibiotic therapy. Further comparison studies and randomized trials are needed to evaluate this concept.

\section{Abbreviations}

CoNS: Coagulase-negative Staphylococci; i.v.: Intravenous; MB: Microbiology; NA: Not applicable; NG: No growth; PM: Polymicrobial

\section{Acknowledgements}

Not applicable.

\section{Funding}

There is nothing to declare.

\section{Availability of data and materials}

The datasets generated and/or analyzed during the current study are not publicly available due the protection of the privacy of subjects involved and ongoing research but are available from the corresponding author on reasonable request.

\section{Authors' contributions}

$\mathrm{PH}$ was responsible for the literary search, patient identification, data collection and analysis, and interpretation and drafting of the manuscript. LL made a substantial contribution to the research design and critical revision of the manuscript. MH made a contribution in the patient identification and critical revision. FH was responsible for the research design, data interpretation, and drafting and revising the manuscript. All authors have read and approved the final submitted manuscript.

\section{Competing interests}

The authors declare that they have no competing interests.

\section{Consent for publication}

Not applicable.

\section{Ethics approval and consent}

This study was approved by the Medical Ethics Research Committee (MERC) of the UMC Utrecht (project number 14-343/C).

Received: 4 November 2016 Accepted: 8 February 2017

Published online: 09 March 2017

\section{References}

1. Darouiche RO. Treatment of infections associated with surgical implants. N Eng J Med. 2004;350:1422-9.

2. Trampuz A, Widmer AF. Infections associated with orthopedic implants. Curr Opin Infect Dis. 2006;19:349-56.

3. Parvizi J, Adeli B, Zimistowski B, Restepo C, Greenwald AS. Management of periprosthetic joint infection: current knowledge: AAOS exhibit selection. J Bone Joint Surg. 2012;94:104.

4. Zimmerli W, Trampuz A, Oschner PE. Prosthetic-joint infections. N Engl J Med. 2004:351:1645-54.

5. Stojicic S, Shen Y, Haapasalo M. Effect of the source of biofilm bacteria, level of biofilm, maturation, and type of disinfecting agent on the susceptibility of biofilm bacteria to antibacterial agents. J Endo. 2013;39(4):473-7.
6. Osmon DR, Berbari EF, Berendt AR, Lew D, Zimmerli W, Steckelberg JM, et al. Diagnosis and management of prosthetic joint infection: clinical practice guidelines by the infectious diseases society of America. Clin Infect Dis. 2013;56(1):e1-25.

7. Worlock P, Slack R, Harvey L, Mawhinney R. The prevention of infection in open fractures: an experimental study of the effect of fracture stability. Injury. 1994;25(1):31-38.

8. Perren SM. Physical and biological aspects of fracture healing with special reference to internal fixation. Clin Orthop Relat Res. 1979;138:175-96.

9. Wehrli W. Rifampin: mechanisms of action and resistance. Rev Infect Dis. 1983;5 Suppl 3:S407-11.

10. Kiedrowski MR, Horswill AR. New approaches for treating staphylococcal biofilm infections. Ann N Y Acad Sci. 2011:1241:104-21.

11. Niska JA, Shahbazian JH, Ramos RI, Francis KP, Bernthal NM, Miller LS. Vancomycin-rifampin combination therapy has enhanced efficacy against an experimental Staphylococcus aureus prosthetic joint infection. Antimicrob Agents Chemother. 2013;57(10):5080.

12. Peck KR, Kim SW, Jung SI, Kim YS, Oh WS, Lee JY, et al. Antimicrobials as potential adjunctive agents in the treatment of biofilm infection with Staphylococcus epidermidis. Chemotherapy. 2003;49(4):189-93.

13. Saginur R, St. Denis M, Ferris W, Aaron SD, Chan F, Lee C, et al. Multiple combination bactericidal testing of Staphylococcal biofilms from implantassociated infections. Antimircob Agents Chemother. 2006;50(1):55-61.

14. Baldoni D, Haschke M, Rajacic Z, Zimmerli W, Tampuz A. Linezolid alone or combined with rifampin against methicillin-resistant Staphylococcus Aureus in experimental foreign-body infection. Antimicrob Agents Chemother. 2009:53:1142-8.

15. Forrest GN, Tamura K. Rifampin combination therapy for non-mycobacterial infections. Clin Microbiol Rev. 2010;23(1):14-34.

16. Mihailescu R, Furustrand Tafin U, Betrisey B, Rio L, Maiolo E, Borens O, et al. High efficacy of fosfomycin-rifampin combination against methicillin-resistant Staphylococcus aureus in an experimental model of foreign-body infection. Clin Microbiol Infect. 2012;18:596.

17. Mouzopoulos G, Kanakaris NK, Kontakis G, Obakponovwve O, Townsend R, Giannoudis PV. Management of bone infections in adults: the surgeon's and microbiologist's perspective. Injury. 2011:42 Suppl 5:S18-23.

18. Widmer AF, Frei R, Rajacic Z, Zimmerli W. Correlation between in vivo and in vitro efficacy of antimicrobial agents against foreign body infections. J Infect Dis. 1990;162(1):96-102.

19. Widmer AF, Gaechter A, Ochsner PE, Zimmerli W. Antimicrobial treatment of orthopedic implant-related infections with rifampin combinations. Clin Infect Dis. 1992;14:1251-3.

20. Zimmerli W, Widmer AF, Blatter M, Frei R, Ochsner PE, Foreign body infection study group. Role of rifampin for treatment of orthopedic implant-related Staphylococcal infections; A randomized controlled trial. JAMA. 1998;279:1537-41.

21. Barberan J, Aguilar L, Gimenez MJ, Carroquino G, Granizo JJ, Prieto J. Levofloxacin plus rifampicin conservative treatment of 25 early staphylococcal infections of osteosynthetic devices for rigid internal fixation. Int J Antimicrob Agents. 2008;32(1):154-7.

22. Drancourt M, Stein A, Argenson JN, Roiron R, Groulier P, Raoult D. Oral treatment of Staphylococcus spp. Infected orthopaedic implants with fusidic acid or ofloxacin in combination with rifampicin. J Antimicrob Chemother. 1997:39(2):235-40.

23. Tschudin-Sutter S, Frei R, Dangel M, Jakob M, Balmelli C, Schaefer DJ, et al. Validation of a treatment algorithm for orthopaedic implant-related infections with device-retention-results from a prospective observational study. Clin Microbiol Infect. 2016;22(5):457.e1-9.

24. Aytac S, Schnetzke M, Swartman B, Herrmann P, Woelfl C, Heppert V, Gruetzner PA, Guehring T. Posttraumatic and postoperative osteomyelitis: surgical revision strategy with persisting fistula. Arch Orthop Trauma Surg. 2014;134(2):159-65.

25. Miller DL, Goswami T. A review of locking compression plate biomechanics and their advantages as internal fixators in fracture healing. Clin Biomech. 2007;22(10):1049-62

26. Schatzker J. Principles of internal fixation. In: Schatzker J, Tile M, editors. The rationale of operative fracture care. 3rd ed. Berlin: Springer; 2005. p. 3-31.

27. Trampuz A, Zimmerli W. Diagnosis and treatment of infections associated with fracture-fixation devices. Injury. 2006;37 Suppl 2:S59-66.

28. Makridis KG, Tosounidis T, Giannoudis PV. Management of infection after intramedullary nailing of long bone fractures: treatment protocols and outcomes. Open Orthop J. 2013;7:219-26. 
29. Achermann Y, Eigenmann K, Ledergerber B, Derksen L, Rafeiner P, Clauss M, et al. Factors associated with rifampin resistance in staphylococcal periprosthetic joint infections (PJI): a matched case-control study. Infect. 2013:41:431-7.

30. Tarrier N, Gregg L, Edwards J, Dunn K. The influence of pre-existing psychiatric illness on recovery in burn injury patients: the impact of psychosis and depression. Burns. 2005;31(1):45-9.

31. Westgeest J, Weber D, Dulai SK, Bergman JW, Buckley R, Beaupre LA. Factors associated with development of nonunion or delayed healing after an open long bone fracture: a prospective cohort study of 736 subjects. J Orthop Trauma. 2016;30(3):149-55.

Submit your next manuscript to BioMed Central and we will help you at every step:

- We accept pre-submission inquiries

- Our selector tool helps you to find the most relevant journal

- We provide round the clock customer support

- Convenient online submission

- Thorough peer review

- Inclusion in PubMed and all major indexing services

- Maximum visibility for your research

Submit your manuscript at www.biomedcentral.com/submit
Biomed Central 